\title{
Cystic Mesothelioma of the Testis in an Adolescent Patient
}

\author{
Alexander J. Chien, MD, Peter J. Strouse, MD, Harry P. Koo, MD
}

Cystic mesothelioma, which is seen primarily in women, usually affects persons in the third to fourth decades of life. We present the case of a cystic mesothelioma in a young man.

\section{CASE REPORT}

A 16 year old African American man was referred with a complaint of sudden onset of right testicular pain, which resolved spontaneously after approximately 6 hours. By the time the patient was seen, he was essentially free of

\section{ABBREVIATIONS}

CT, Computed tomography

Received December 3, 1999, from the Section of Pediatric Radiology, C.S. Mott Children's Hospital, Department of Radiology (A.J.C., P.J.S.), and the Section of Urology, Department of Surgery (H.P.K.), University of Michigan Health System, Ann Arbor, Michigan. Revised manuscript accepted for publication March 20, 2000.

Address correspondence and reprint requests to Peter J. Strouse, MD, Section of Pediatric Radiology, C.S. Mott Children's Hospital-F3503, University of Michigan Health System, 1500 E. Medical Center Drive, Ann Arbor, MI 48109-0252. pain. At its most severe degree, the young man described the pain to be 7 on a scale of 10 . He denied any history of trauma, fever, nausea, penile discharge, or voiding symptoms. Physical examination was notable for bilateral cremasteric reflex. The left testis was normal in size and contour. The right testis was surrounded by multiple soft nodularities, which were minimally tender to palpation.

Ultrasonographic examination confirmed the presence of an abnormality within the right hemiscrotum (Fig. 1). A hydrocele was noted with particulate debris within the fluid. Within the hydrocele fluid were multiple, discrete, small cystic structures measuring less than $10 \mathrm{~mm}$ in size and containing clear fluid. Doppler interrogation confirmed that the cysts were not vascular structures. The testes appeared normal and symmetric on gray scale and Doppler evaluation.

Right inguinal exploration was performed with intraoperative pathologic evaluation of frozen tissue. Upon delivering the right testis and incising the tunica vaginalis, approximately $5 \mathrm{ml}$ of hydrocele fluid was drained. The inner aspect of the tunica vaginalis was found to be studded with small cysts ranging from $2 \mathrm{~mm}$ to $2 \mathrm{~cm}$ in diameter (Fig. 2). In addition, several small cysts were noted to be adherent to the tunica albuginea of the testis. Pathologic evaluation of several frozen section specimens was consistent with benign cystic mesothelioma. With the report of benign tissue, the decision was made to excise the cysts and preserve the testis.

The patient had an unremarkable early postoperative course. A CT examination showed no evidence of intraperitoneal cysts. A testicular sonogram at 4 months after surgery revealed testes of normal size bilaterally with 


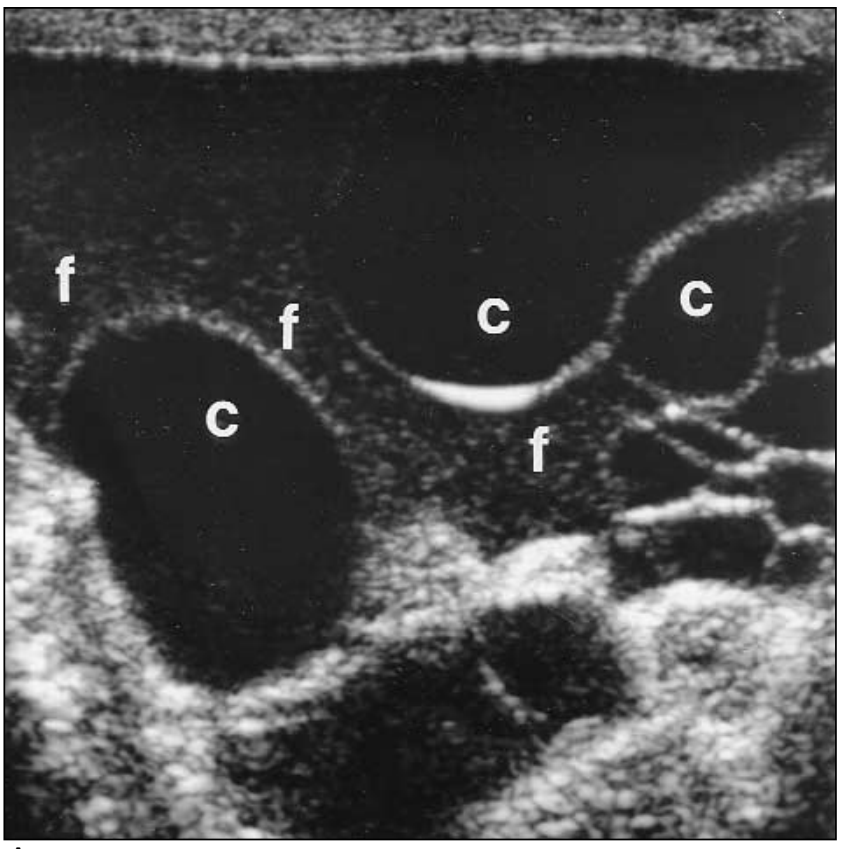

A

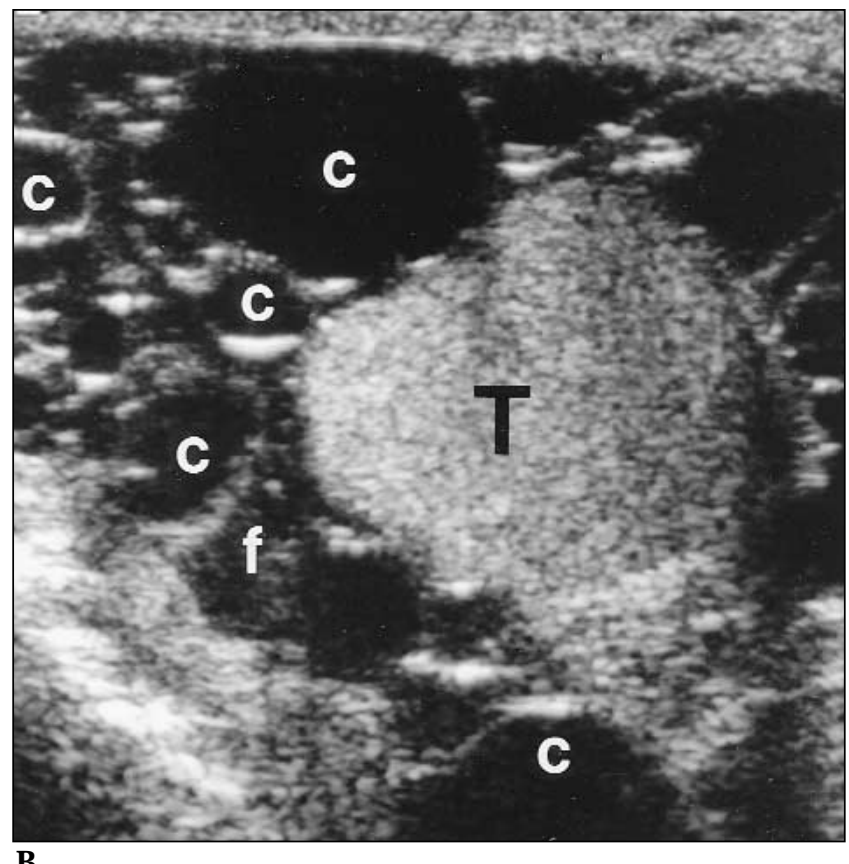

B

Figure 1 A, B, Sonogram of the right hemiscrotum. Hydrocele fluid (f) is present and contains particulate debris. This fluid was seen to flow freely at real-time examination. Multiple small cysts (c) are seen. T, Right testicle.

a $3 \mathrm{~mm}$ cyst at the inferior aspect of the right testis. Although the patient remained asymptomatic, repeat sonograms at 1 and 2 years after surgery showed recurrence of the cystic mesothelioma with a gradual increase in both the number and size of cysts within the right hemiscrotum. At 2 years after the initial surgery, the patient underwent definitive right inguinal orchiectomy.

\section{DISCUSSION}

Cystic mesothelioma is a tumor primarily of young to middle-aged women with a 5:1 female-to-male predominance.1,2 About one half of the cases occur the third to fourth decades of life (mean age, 38.1 years). ${ }^{1,2}$ These tumors affect chiefly the pelvic peritoneum. ${ }^{1}$ No consistent etiologic factor has been identified for peritoneal cystic mesothelioma; however, an association with prior laparotomy appears to exist.1,2 We were unable to locate any previous reports of a cystic mesothelioma of the scrotum, although scrotal malignant mesothelioma has been reported previously.3,4 Stein and coworkers did report a case of a noncystic mesothelioma of the tunica vaginalis of a child. 5

Patients with peritoneal mesothelioma most commonly have presenting symptoms of abdominal pain and a tender mass. ${ }^{1,6,7}$ Ultrasonographic findings of peritoneal cystic mesothelioma have been described previously as a multicystic mass.7-9 On laparotomy, such tumors have been described as transparent, with thin-walled cysts and grapelike, fluid-filled nodules or clusters. $1,8,9$ Similar findings were evident in our case.

The sonographic features of the lesion in our patient were distinctive. Although a number of pathologic processes could account for fluid in the scrotum (infection, trauma, simple hydrocele), the differential diagnosis for multiple small extratesticular cysts is extremely limited. Spermatoceles or epididymal cysts may be multiple, but usually not to the degree seen in our patient. Moreover, such lesions should be confined to the epididymis. Papillary cystadenomas of the epididymis, associated with von Hippel-Lindau disease, often are cystic; however, they also should be confined to the epididymis. 10 A hematocele or pyocele may have septations or loculations, creating the appearance of multiple cysts; however, usually the clinical history or physical examination will suggest the proper diagnosis. Varicoceles might be mistaken for cysts; however, their location (posterior or superior), serpiginous appearance, and evidence of blood flow on Doppler interrogation should suggest the proper diagnosis. Malignant mesothelioma of the tunica vaginalis may produce a hydrocele; however, solid nodular implants or irregular thickening of the tunica vaginalis is seen as opposed to cystic lesions. 3,4 
The prognosis of cystic mesothelioma in the peritoneal cavity is excellent. Little, if any, correlation exists between the extent of tumor and patient survival. ${ }^{1}$ Of 25 patients reported by Weiss and Tavassoli, ${ }^{1}$ with a follow-up time from 5 months to 31 years, two patients died of the disease (one infant and one patient who refused therapy). ${ }^{1}$ Local recurrence is common. In a series reported by Ross and colleagues, ${ }^{9} 15$ of 23 patients with cystic mesotheliomas treated by incomplete resection had recurrence on follow-up evaluation at 6 months to 17 years after surgery. Only one of seven patients undergoing complete resection had recurrence. ${ }^{9}$ Spread within the peritoneum, apart from local recurrence, is rare. Despite the tendency for local recurrence, cystic mesotheliomas have not shown evidence of malignant degeneration or distant metastases, $1,2,9$ and morbidity therefore has been attributed to the local effects of the tumor. $1,2,6-10$

Therapy for cystic mesothelioma of the peritoneum has usually been either total or subtotal resection.1,2,6-10 Although adjunctive radiotherapy, intraperitoneal chemotherapy, and local sclerosing therapy has been attempted, many authorities believe that radiation and chemotherapy are contraindicated in this benign disease. ${ }^{1}$ Many patients have required repeated surgical resection. 1,9

We present a case of a rare benign tumor, even more unusual because of the age and sex of the patient and the site of presentation. The lesion had a novel ultrasonographic appearance. On the basis of the sonographic and clinical evidence of recurrent disease and our review of the literature on peritoneal tumors of this type, we decided that a unilateral orchiectomy was indicated to eradicate the tumor and prevent further recurrence.

Figure 2 Operative photograph of the opened right hemiscrotum. Multiple small cysts are seen, correlating with the sonographic findings. T, Right testicle.

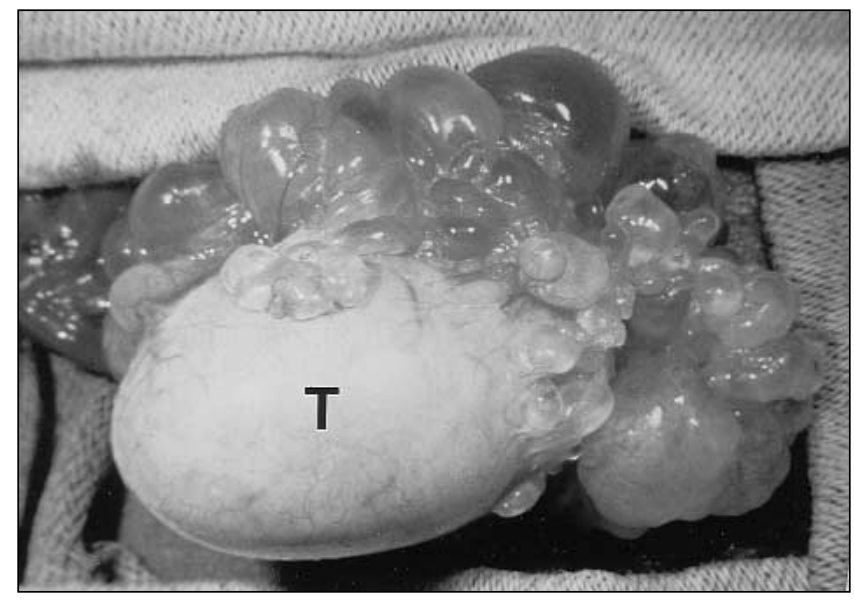

\section{REFERENCES}

1. Weiss S, Tavassoli F: Multicystic mesothelioma: An analysis of pathologic findings and biologic behavior in 37 cases. Am J Surg Pathol 12:737, 1998

2. Saint-Paul MT, Salles IT, Bailly C, et al: Le mésothéliome multikystique péritonéal bénin: A propos d'une observation. Revue de la littérature. J Gynecol Obstet Biol Reprod 21:30, 1992

3. Tyagi G, Munn CS, Kiser LC, et al: Malignant mesothelioma of tunica vaginalis testis. Urology 34:102, 1989

4. McDonald RE, Sago AL, Novicki DE, et al: Paratesticular mesotheliomas. J Urol 130:360, 1983

5. Stein N, Henkes D: Mesothelioma of the testicle in a child. J Urol 135:794, 1986

6. Canty M, Williams J, Volpe R, et al: Benign cystic mesothelioma in a male. Am J Gastroenterol 85:311, 1990

7. Takenouchi Y, Oda K, Takahara O, et al: Report of a case of benign cystic mesothelioma. Am J Gastroenterol 90:1165, 1995

8. McCullagh M, Keen C, Dykes E: Cystic mesothelioma of the peritoneum: A rare cause of "ascites" in children. J Pediatr Surg 29:1205, 1994

9. Ross M, Welch W, Scully R: Multilocular peritoneal inclusion cysts (so-called cystic mesotheliomas). Cancer 64:1336, 1989

10. Price EB Jr: Papillary cystadenoma of the epididymis: A clinicopathologic analysis of 20 cases. Arch Pathol 91:456, 1971 